\title{
STATIC COMPONENT INTERCONNECTION TEST TECHNOLOGY IN PRACTICE
}

\author{
Frans de Jong, Rob Raaijmakers \\ Philips Research, Philips CFT, The Netherlands \\ Email: frans.de.jong@philips.com,r.m.w.raaijmakers@philips.com \\ Steffen Hellmold \\ Fujitsu Microelectronics Inc., USA \\ Email: steffen.hellmold@fmi.fujitsu.com
}

\begin{abstract}
Static Component Interconnection Test Technology (SCITT) is a new XNOR circuit based technology that is used for board-level interconnection test.

SCITT provides an easy test method using simple patterns and results in a high diagnostic resolution. The method is especially suited for SDRAM and other 'complex memories' but can be used for other devices as well. Very little overhead is required. A real silicon implementation is presented and evaluated.
\end{abstract}

\section{Introduction}

Modern designs have increasing functionality resulting in increasingly complex designs. The high component density of the designs and the use of package types like Ball Grid Arrays (BGA) for the ICs make verification of first prototypes difficult. If a simple test can weed out the assembly failures quickly, verification can focus on the functional aspects. For volume production, the main focus of assembly test is to check the structure of the assembly. Many test methods are hampered by the lack of access for signal measurements by the use of the modern technologies. Therefore, electronic access, as provided by Boundary Scan is an increasingly popular method. For some components Boundary Scan is not suited or not possible. One class of such components is the so-called 'complex memories'. For these components, this paper introduces the concept of SCITT. SCITT stands for Static Component Interconnection Test Technology and uses the functionality of XNOR gates to replace the functionality of the complex memories during testing.

Sections 2 and 3 introduce this concept in more detail. A typical application area is shown in section 4, followed by implementation remarks on different memory types in section 5 . In section 6, test mode control is discussed along general points of attention. A description file is introduced in section 7, just before we start the discussion on a real example in sections 8,9 and 10 . Section 11 deals with some comparative calculations on different but related test ideas. Finally some remarks on Design for Test (DfT) with respect to SCITT are given in section 13, followed by the concluding remarks. The internal details on the XNOR circuits can be found in an accompanying paper [2].

\section{What is SCITT}

SCITT stands for Static Component Interconnection Test Technology and requires the insertion of XNOR circuits in a device. These XNOR circuits (see figure 1) replace the normal function of a chip when in test mode. It is basically meant for board-level test.

The acronym SCITT shows two important aspects:

1. It is about 'static' testing. Once in test mode it bypasses the dynamic (parametric and high frequency) properties of the component.

2. It is about component interconnection testing, verifying the connections between the device and its surroundings.

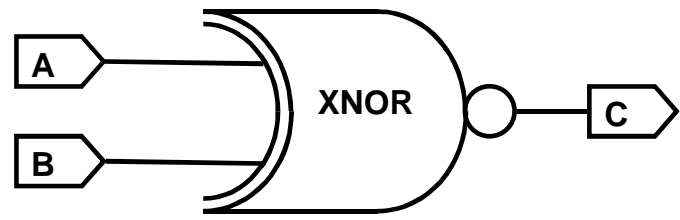

\begin{tabular}{l|cc|c|}
$\begin{array}{l}\text { Truth } \\
\text { table }\end{array}$ & A & B & C \\
\cline { 2 - 4 } & 0 & 0 & 1 \\
0 & 1 & 0 \\
1 & 0 & 0 \\
1 & 1 & 1 \\
\hline
\end{tabular}

Figure 1: XNOR function 
SCITT is developed to provide a cheap test solution in combination with Boundary Scan [1]. It is therefore not a substitute for but an addition to Boundary Scan as test solution.

It is assumed that the device, which has this test circuitry implemented, can be fully accessed on all its pins at board level. This can be realised, amongst others, by access from an adjacent Boundary Scan device or from direct access from an edge connector. This is illustrated in Figure 2.

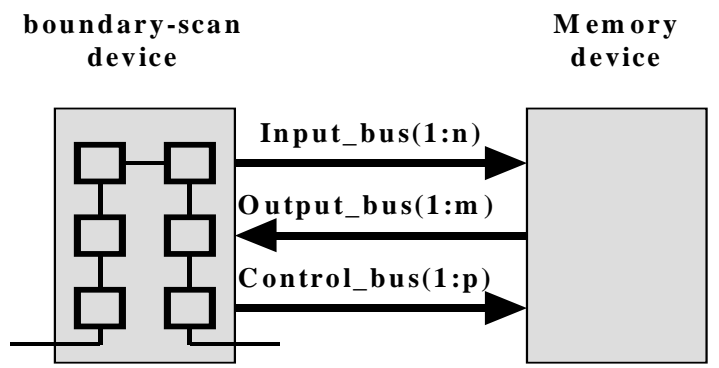

Figure 2: Boundary Scan access to a memory device

The basic idea of SCITT is simple: consider all outputs of a device as outputs of XNOR circuits. Each output function (pin) must have a unique mapping on the inputs of the device. Furthermore a fail-safe way to get in and out of test-mode is required. The schematic block diagram in figure 3 shows how to prevent entering the functional block (in this case the SDRAM core) in test mode.

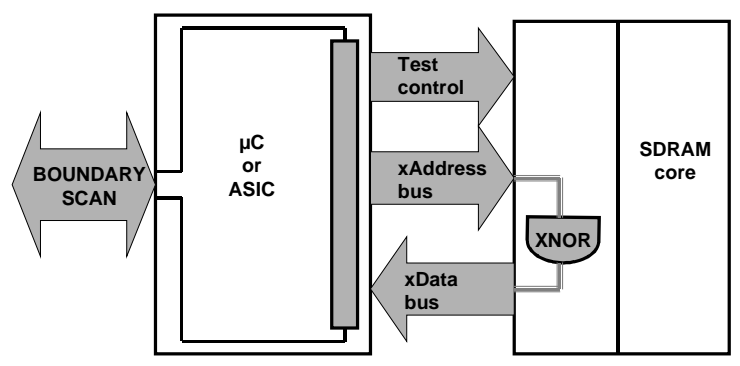

Figure 3: Test access and location of implementation

In test mode a simple, static, logic (XNOR) function remains that replaces the original function of the device, as 'seen' from it's pins. This logic function enables easy detection of all single stuck-at and bridging faults that may occur during assembly.

\section{Some basics}

A memory device has three groups of connections, a control bus, an address bus and a data bus (see figure 2).
From the control bus a minimal but necessary sub set is selected for SCITT test control. The remaining control connections are combined with the regular inputs (address bus) and together they form the set of extended inputs for the SCITT circuitry. When control outputs are present as well, they are combined with the regular outputs (data bus) to form the set of extended outputs. In this way, for SCITT there is always only a set of extended inputs, connected through the XNOR functions to a set of extended outputs. Note that the (data) outputs may have a bi-directional functionality. It is stated that the proof of a correct interconnection need only to be made in a single direction. The capability to check the correct functioning of the total I/O buffer after assembly is lost with this method.

To maximise diagnostics, each XNOR function must have an odd number of inputs greater than one and each output must have a unique combination of inputs connected [2]. In test mode, SCITT changes the 'complex' memory function into a simple logic cluster containing XNOR functions.

In order to keep test pattern generation simple, the basic set of a walking ' 1 ' and walking ' 0 ' sequence is applied to the extended set of inputs. Additionally, an all ' 1 ' and all ' 0 ' pattern complete the set of stimuli. So, the number of used test vectors is also very small. This is a requirement for this technology if it is used with Boundary Scan. If $\mathrm{N}$ is the number of extended inputs the total number of test vectors is $2 \mathrm{~N}+2$, which is linear with the number of inputs of the memory devices.

\section{When to use SCITT}

In modern multi media designs, basic elements are mixed signal I/O, a processor and some memory (see figure 4). Memory and mixed signal circuits often lack a test mode that is "compatible" with Boundary Scan. Trends in modern designs also show that processor speed and the amount of memory connected are still increasing. Also more and more "complex memories" are being used. A complex memory is a memory with an embedded protocol or memories that need initialisation before use. Examples are SDRAM, FCRAM, RAMBUS, FLASH, and FIFOs.

For the memory market, price and pin compatibility are very important. The silicon overhead and four extra pins needed to implement Boundary Scan are unacceptable.

The protocols and initialisations, together with dynamic access restrictions for the memories, make interconnect testing by means of Boundary Scan from surrounding devices difficult. The difficulty is especially influenced by the Boundary Scan chain length involved (TriMedia has 385 cells). Even with special algorithms [3][6], the dynamic memory timing aspects of SDRAM, FCRAM or RAMBUS memories are still to be respected. If a connection is failing, proper access to the devices may be impossible at all, leaving this test option meaningless. 
SCITT can overcome these problems because of its static

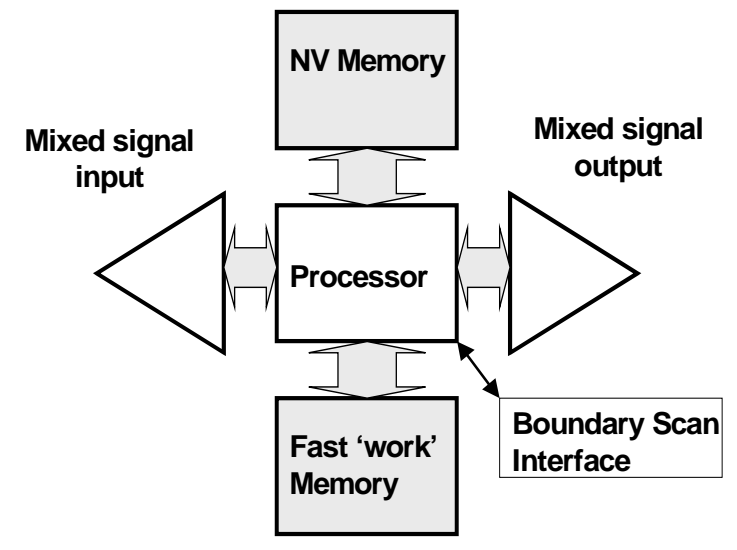

Figure 4: Generic multi media block diagram.

nature during test. SCITT needs no or almost no extra pins and requires very little silicon overhead. The first areas of interest for SCITT must be these price driven memory markets with standardised packages, no defined test pins on the package and dynamic restrictions.

\section{A look at some memories}

\section{- FLASH}

The long erase time of FLASH memories after an interconnect test make such a test, before in-line final programming, not economical. The use of pre-programmed flash devices on the assembly line is expensive as well, mainly due to logistics. With a SCITT test mode there is no programming involved, so erasure is not needed and preprogrammed flash devices may be used on the assembly line. Alternatively, in-line downloading can now be done in proven correctly connected devices.

The byte/word input control needs special attention for SCITT application. Sometimes this pin is strapped to a fixed value which may imply that part of the outputs ('high byte') is not used.

In general two issues are important:

1. With strapped pins, the tests of other pins and diagnosis of the results should not be hampered due to a wrongly chosen combination of inputs on the XNOR circuits.

2. All of the inputs must, at least, map on the smallest set of outputs, as defined in the functional description (like 'byte mode'). However, a valid SCITT implementation must also be implemented on the remaining outputs.

3.

- FIFO

Testing interconnects of deep FIFOs may take a lot of time when done with a Boundary Scan approach because of the 'half-full' and 'full' flag signals. They must be tested and that involves many write cycles. Because of the decoupling of function and actual contact to a device pin, this test can be minimised using SCITT.

In figure 5 an implementation diagram for FIFOs is shown. Again, for test control only a few lines are needed. The remaining control in- and output signals are added to the sets of extended inputs and outputs, respectively. The idea is that test control can be realised with an arbitrary set of control lines that does not influence the functional behaviour. Additionally, during functional mode, there should not be a combination of control signals which can inadvertently put the device in test mode.

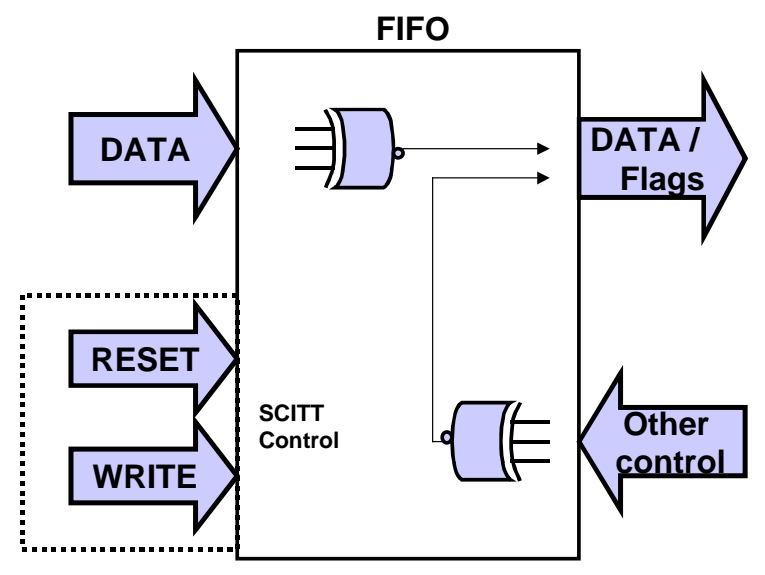

Figure 5: SCITT implementation proposal for FIFO

\section{- SDRAM}

For SDRAM, an implementation will be discussed in more detail from chapter 8 onwards.

\section{Test mode control}

Finding a possible solution for the test problem is only half the answer. The test should be flexible, controllable and not brand or type dependent. This makes standardisation of the access methods and a description of the XNOR circuit implementation necessary. Because of the existence of many different complex memory types, the description for test mode control can only be given along general lines:

- Test mode entry must be possible at power up. This is because interconnect testing is one of the first things to be done.

- Test mode control must make it possible to enter or resume normal operation. This is because many follow up tests require power and a functioning memory as well. A power down followed by a power up sequence can take a lot of time.

- Use as few control pins as possible to get a robust test mode control. This is because the test mode control pins are harder to diagnose when failing. 
- Glitches on the control pins during functional mode may put the device in test mode. The device must either return automatically to functional mode or a normal reset procedure should work.

- Define a test mode control sequence that can be described and interpreted unambiguously. This is to make automatic control (pattern) generation possible.

- When applicable, an extra test state in the devices' state diagram might be added, which is also addressable from functional mode. This makes a software based self-test of a system easier.

- (Optional) When possible implement the test control using one (or more) additional test pin(s). Such a pin is the best means for control of SCITT mode (but still more expensive).
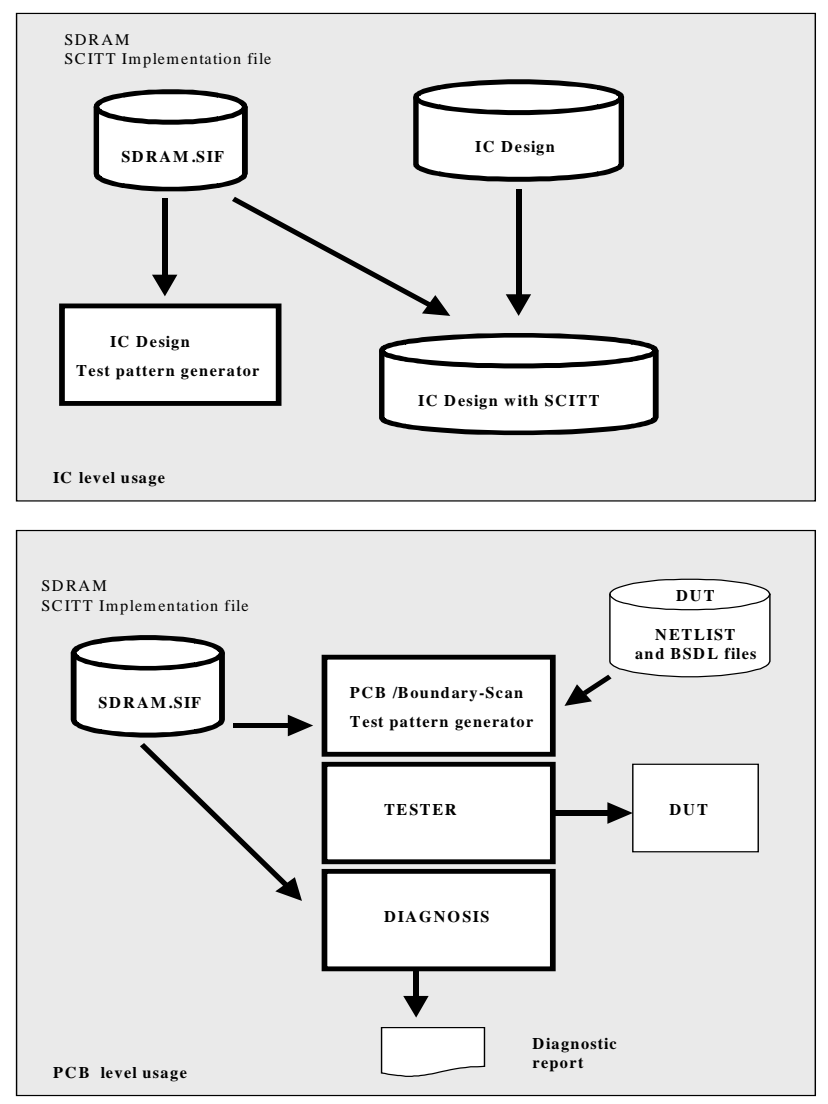

Figure 6: SCITT Description file used at IC and assembly level.

\section{The SCITT description file}

Just like the BSDL file for Boundary Scan, a description file for SCITT is required.

This file should contain at least the following aspects:
- The XNOR circuits must be given, including their connections to physical pins.

- The test mode control sequence must be given, which describes the signal levels to apply after power up to enter SCITT mode.

- $\quad$ The sequence of signal levels to exit this test mode.

Further details are possible in an optional section of this file. Options like the availability of a dedicated test pin (one or more), or the description of an added test state to a state diagram are possible. Also a set of test patterns may be included (see appendix A). A proposal for standardisation is to write these implementation details in Verilog[7]. Verilog was used for the definition of the circuits for implementation and for the simulation of the circuit as implemented in an SDRAM. Currently work is ongoing for the exact definition of the contents of this file. Due to the simplicity of the pattern generation and electronic distribution of datasheets it is thought best to distribute the design description as part of the device datasheet. The usage of this file, after extraction from the datasheet, can be three fold:

1. For the specification of the implementation for generating the circuits during IC design.

2. For generating the actual test patterns automatically (two times: for IC test as well as for the actual board assembly test).

3. During the diagnostic process. A program may find out what went wrong, based on the test results and this description file.

The usage of this file is depicted in figure 6. This figure shows two separate processes. The upper part depicts IC design and testing. The lower part depicts PCB assembly test.

\section{A SCITT testable SDRAM example}

The initial idea for SCITT came up when testing a board containing an advanced processor and some SDRAM devices, similar to those in figure 7 .

The main problem is that the SDRAMs have an internal state-machine and registers that are controlled by almost all its address lines. One single solder error on these lines can leave the system dead because the control of the MRS register value is lost through bridge errors, stuck at faults or opens. For this example a demo-board with a Philips TriMedia processor and Fujitsu 64Mbit (2Mx32) MB81F643242B SDRAM is used [4].

To implement SCITT in a SDRAM requires an extra test state. This state is added in such a way that it can only be entered directly after the power-up sequence (see figure 8). 


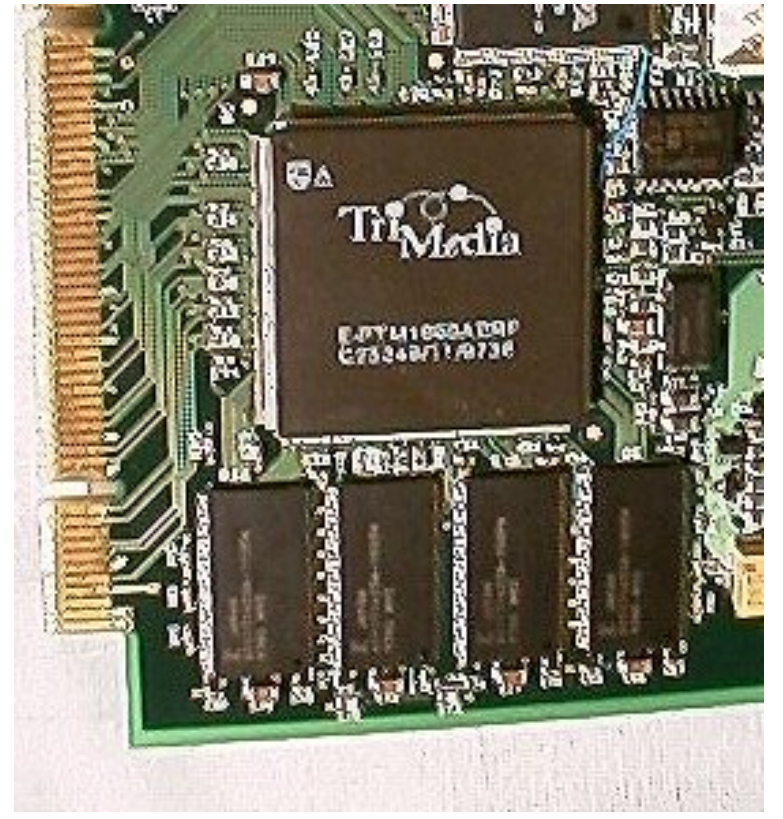

Figure 7: Typical SCITT application area on a multi media design.

The main reason for this is functional safety, robustness and backward compatibility.

Checking the controller definition in the SDRAM for use of control lines showed a possibility to enter the test-mode without extra pins. Only three control lines, CSn, CASn and CKE are needed.

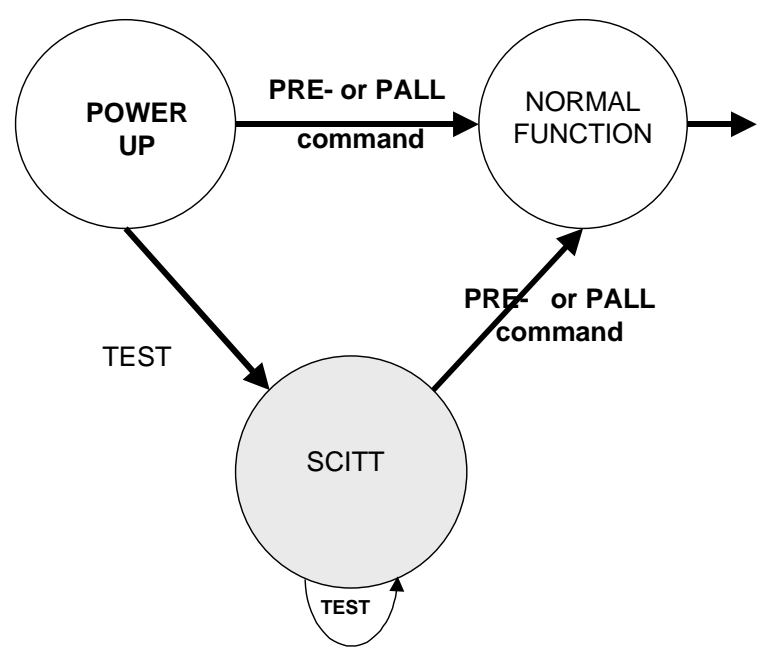

\section{Figure 8: SDRAM state-machine extension}

The SDRAM enters test-mode with a high to low level transition on the CASn signal while CSn and CKE are low after power-on (see figure 9).

In test mode, the CSn signal determines which device is selected for testing. This normal function allows separate device or bank switching if multiple memories are connected. The CKE signal is used to enable (high) or disable (low) the defined test output signals.

The device will exit test mode if the CASn line is set high. When leaving the test mode, the normal initialisation sequence (apply 'precharge' command) can be continued. This is exactly the expected normal behaviour and therefore the controller won't notice the difference even if the device enters test mode by accident.

Except for the three control signals ALL remaining signals become input or output of the device in test mode (see table 1).

Table 1:Test mode signal allocation

\begin{tabular}{|l|l|l|}
\hline control & Inputs & outputs \\
\hline CSn & RASn & DQ(0..31) \\
\hline CASn & $\mathrm{A}(0 . .12)$ & \\
\hline CKE & DQM $(0 . .3)$ & \\
\hline & CLK & \\
\hline & WEn & \\
\hline
\end{tabular}

Because no extra pins are used to enter or exit test-mode, the package is pin-compatible with existing JEDEC standards. The actual signal levels required for SCITT control are shown in figure 9.

Re-entry of the test-mode after power up is not possible. As soon as a precharge command is applied the test-modeentry command is disabled. This implementation was

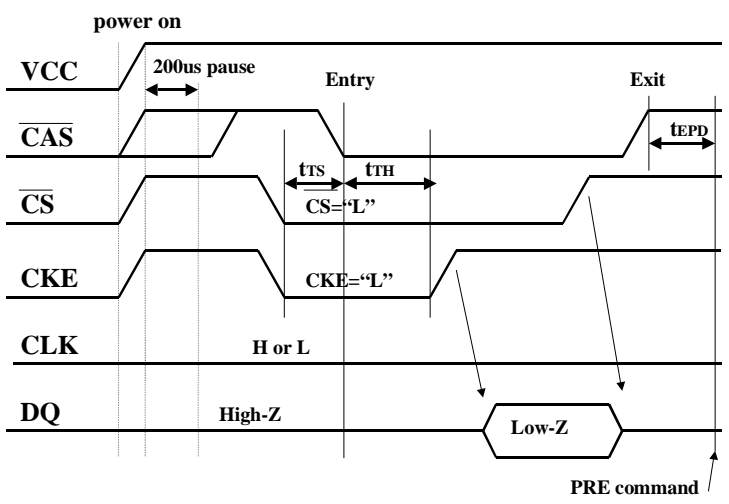

Figure 9: SCITT control signals at power up.

chosen to prevent the device entering test mode while in normal operation since the test-mode entry command sequence might occur during normal operation of the device.

\section{SDRAM, a first implementation}

When the SDRAM is in test mode the chip has three control lines, 20 input, and 32 outputs. The Verilog file 

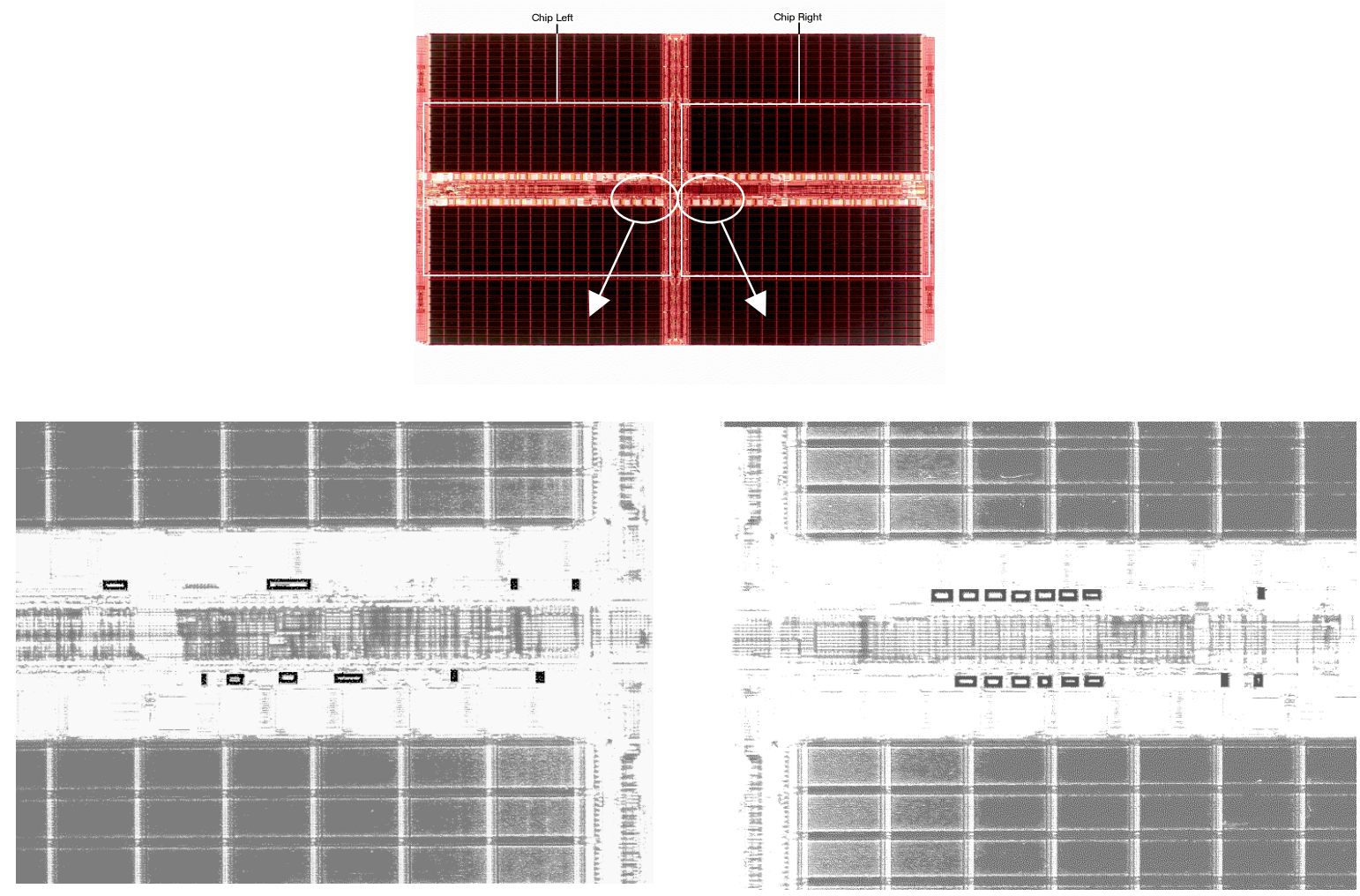

Figure 10: Photographs of sections of the B-version (SCITT = black spots) of the Fujitsu die. The small picture on top is an overview of the whole chip.

(Appendix B) shows the actual description of the 32 XNOR circuits and their connections to the pin numbers. This is an early implementation with two-input XNORs. Although $100 \%$ coverage is achieved, which is responsible for detection, it may show slightly less diagnostic capabilities than a realisation with three-input XNORs [2]. Detailed calculation shows a slight increase of the access time by only $0.05 \mathrm{nsec}$. The die size penalty for this first implementation was about $0.3 \%$. When implemented from the beginning of a design cycle and not as final addition, this silicon increase could even be less than $0.1 \%$.

The photograph of the die shows the area of SCITT implementation (figure 10). Notice the small black rectangles that indicate the SCITT implementation areas

\section{Results from practice}

Verilog simulations on the Fujitsu SDRAM model done at Philips showed that entry and exit of test mode function correctly. The actual tests on the real device showed correct behaviour as well.

The 20 inputs will result in a total of 42 test patterns (see appendix A) needed to detect all stuck at and bridging errors for one chip on an assembly.
Fault simulations done on the implemented two-input XNOR circuits show that the fault coverage on the stuck at and bridging errors is indeed the expected $100 \%$ on the extended inputs and extended outputs.

Table 2: Test coverage and diagnostic resolution for SDRAM circuit 'en20_32'.

\begin{tabular}{|l|l|}
\hline \multicolumn{1}{|c|}{ Circuit: } & $\begin{array}{l}\text { En20_3 } \\
2\end{array}$ \\
\hline Coverage stuck-at 0/1 input pins & $100 \%$ \\
\hline Coverage stuck-at 0/1 at all output pins & $100 \%$ \\
\hline Coverage wired-and bridges & $100 \%$ \\
\hline Coverage wired-or bridges & $100 \%$ \\
\hline
\end{tabular}

One of the improvements for board-level test is that strapped pins are detected as such and will not block the circuit as was encountered using a NAND-tree [5].

The silicon implementation is tested with a board using a Philips TriMedia DSP (TM1100) as SDRAM controller with Boundary Scan and two 8MByte Fujitsu MB81F643242B SDRAM devices. 
The SDRAM is tested in normal function at $100 \mathrm{MHz}$ and $120 \mathrm{MHz}$. No functional flaws were detected.

Figure 11 shows the Boundary Scan set up as used for verification. The only Boundary Scan device in the chain is the TM1100 with a register length of 385 cells. All

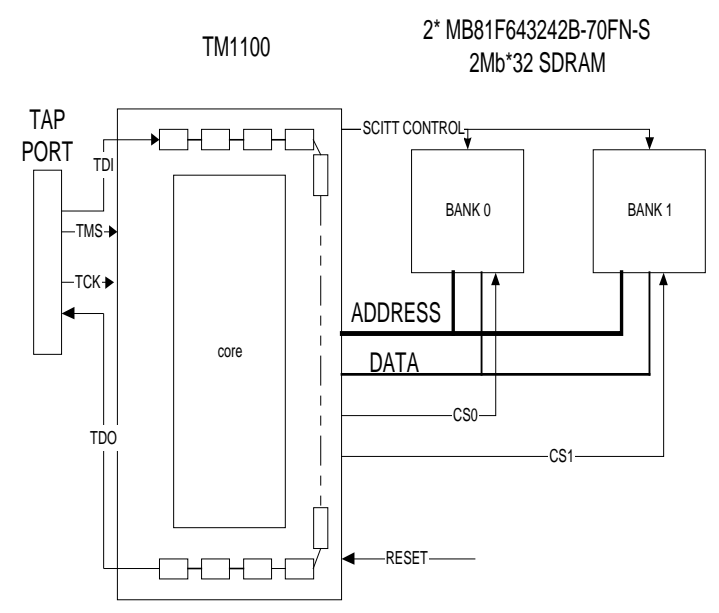

Figure 11: boundary-scan chain set up

address, data and control lines of the SDRAM are connected to the TriMedia processor and have full Boundary Scan access. A total of 46 test patterns are applied for test: 42 patterns are needed for the coverage (see appendix A), three additional patterns are needed for test mode entry, and one is needed for test mode exit. The actual test execution took $412 \mathrm{~ms}$ with a $43.7 \mathrm{KHz}$ tester (PC printer port based tester). This matches the calculated test time very well.

The 'normal' memory test for the demo-board consists of a program download using the JTAG-debug port of the TriMedia that starts running a $12 \mathrm{~N}$ test algorithm. This test takes 30 seconds, and an optimised version still takes 5 seconds. A general remark is that this type of testing also checks for the internals of the memories and is interesting for prototype testing. This is however not required (and time consuming) during PCB assembly testing. Calculations indicate that a Boundary Scan test with a SCITT device, on a $10 \mathrm{MHz}$ tester (TCK clock speed) only takes about $3.4 \mathrm{msec}$. This improvement of more than a factor 1000 is considerable but merely applicable for prototype testing.

\section{Comparison of four methods}

Comparison of different test methods for SDRAM. To make a comparison all options are calculated with a processor having a Boundary Scan cell length of 385 and 2 SDRAM devices of $4 * 512 \mathrm{~K} * 32$ organisation. The Boundary Scan state machine control clock cycles are neglected.
1- Boundary Scan on the SDRAM.

First the estimated Boundary Scan implementation for SDRAM is given. 32 bidirectional data pins, using a 2-cell implementation result in 64 cells. The 12 address pin inputs, realised with a 1-cell implementation results in 12 cells. Finally, the 11 control pins use also a 1-cell implementation, resulting in 11 cells. This makes a total of 87 cells per device. The total chain length of the test set up is: $385+87+87=559$.

Secondly, for the test patterns about 16 patterns are required: three possible drive sources on data lines that drive stuck-at patterns (all ' 0 ' and all ' 1 ') result in six patterns, ten patterns are required for a 'counting sequence' and its inverse set [8]. This sums up to a total of 16 patterns. The total number of test clocks necessary is $559 * 16$ = 8944. Required test time (TCK @ $10 \mathrm{MHz})$ : $0.9 \mathrm{msec}$.

\section{2- SCITT implementation.}

In this case, the Boundary Scan chain length (controller only) is 385 cells.

Required test vectors: 3 for SCITT entry, 84 patterns for test of two devices and 1 for exit. This makes a total of 88 vectors. The total number of test clocks necessary is $385 *$ $88=33880$. Required time (TCK @ $10 \mathrm{MHz}$ ): $3.4 \mathrm{msec}$ Note: with an extra test pin on the SDRAM the initialisation (entry) would not be necessary.

3- Functional test approach using Boundary Scan. During this functional test, the Boundary Scan tester or controller must emulate the normal cycles on the SDRAM. Initialisation after power-on: 27 states to active idle state (PALL, 8*[IDLE, REFRESH, PALL] IDLE.). For an optimised test vector set that includes the CAS latency of 2, we need 6 vectors per write and 8 vectors per read cycle. As used for memory test, 24 address line and 6 data line patterns are necessary [3][6]. The 24 address line checks need a write-write-read cycle. This makes $24 *(6+6+8)=$ 480 vectors. The data line check are simple write-read cycles and require $6 *(6+8)=84$ vectors. This makes a total of 564 vectors necessary for the test per chip. The total number of test clocks necessary for two devices is $2 * 385 *$ $564=434280$.

Required time (TCK @ $10 \mathrm{MHz}$ ): $43.4 \mathrm{msec}$

Note1: to remain within the timing limits of the SDRAM specification, the test speed (TCK) must be calculated in relation to the chain length and the most critical time involved (like e.g. RAS pulse width).

Note2; solder errors on the address lines can lead to illegal or invalid states in the state-machine of the SDRAM.

Behaviour of the state-machine, especially under fault conditions, is vendor dependent. All this may make the diagnosis quality unpredictable or even impossible. 


\section{4- Full functional}

Without using Boundary Scan, we may use normal software that executes the same test as mentioned under 3. If the test passes it is much faster but depends on the interface speed. The number of patterns is only 168 ( 24 times writewrite-read and $6 *$ write-read per device). Required time (uP @ $100 \mathrm{MHz}$ ): $0.2 \mu \mathrm{sec}$

In case of faulty connections, strange behaviour can be expected, even system crashes are observed.

Next to the diagnostic restrictions that are the same as in 3 , additional requirements for board level are:

- The test software must be available (ROM code or downloadable).

- The processor must run without using the SDRAM under test for program execution.

- The processor must run even with failures (bridges) on its SDRAM connections.

- For control and diagnostics, an access channel through the processor is needed.

\begin{tabular}{|c|c|c|c|}
\hline \multirow{3}{*}{$\begin{array}{l}\text { Method: } \\
\text { 1. Full Boundary } \\
\text { Scan }\end{array}$} & \multicolumn{2}{|c|}{ PatternsTest time } & Diagn. \\
\hline & 16 & $0.9 \mathrm{msec}$ & ok \\
\hline & 88 & $3.4 \mathrm{msec}$ & ok \\
\hline 3. Emulated functional & 1128 & $43.4 \mathrm{msec}$ & poor \\
\hline 4. Functional, at speed & 1128 & $0.2 \mu \mathrm{sec}$ & poor \\
\hline
\end{tabular}

\section{Remarks on Design for Test}

At the board level, a few details need to be taken care of to use SCITT.

- The controlling processor needs to have a reset or halt capability that can be held active during the power up phase. This is required to prevent the processor from inadvertently initialising the SDRAMs.

- An access point or test spot is required on this signal.

- The controlling processor must preferably have Boundary Scan implemented.

- ALL lines of the SCITT memory must be accessible.

\section{Remarks and conclusions}

The use of SCITT is not limited to board-level test. Manufacturers of the IC's can also use SCITT effectively for among others IC bond-wire testing and IO parametric tests.

Fujitsu designers estimate that the SCITT logic could be hidden in the pad area in case of a new design so the die size increase is almost zero. For a re-design of an existing chip which is the case for the Fujitsu MB81F643242B, the silicon overhead proved to be less then $1 \%$.

The implementation considers all bi-directional data pins as outputs. This limits the effective check of the related input buffers. At board level, the target is set to interconnect tests, therefore this is not a problem. However, improvements are desirable because potential assembly damage should be covered as early as possible in the test process as well.

This work is still in progress and a definition that can be seen as a standard is one of the focal points of current activities. At the time of writing, we are working on standardisation within JEDEC as this will bring the optimal results for the test community.

\section{Acknowledgements}

This work is made possible with partial funding of the European MEDEA programme (project A101/A222).

\section{References}

[1] IEEE Test Technology Technical Committee of the IEEE Computer Society, 'IEEE Std. 1149.1 Standard Test Access Port and Boundary-Scan Architecture', Institute of Electrical and Electronic Engineers, October 21, 1993, ISBN 1-55937-350-4.

[2] Biewenga A.S.B., Hollmann H.D.L., De Jong F.G.M., Lousberg G.E.A, 'Static component Interconnect Test Technology (SCITT), a new technology for assembly testing', Paper submitted for ITC99.

[3] Biewenga A., Muris M., 'Using Boundary Scan Test To Test Random Access Memory Clusters', IEEE Proceedings of the International Test Conference, 1993, pp 174-179.

[4] Fujitsu SDRAM datasheet MB81F643242B Version AE0.1E.

[5] Robinson Gordon D., 'NAND Trees Accurately Diagnose Board-level Pin Faults', IEEE Proceedings of the International Test Conference, 1994, pp 811816.

[6] De Jong F., A., De Lind van Wijngaarden A., 'Memory interconnection test at board level', IEEE Proceedings of the International Test Conference, 1992, pp 328-337.

[7] The Verilog Hardware Description Language (HDL), IEEE Std. 1364-1995. 
[8] Jarwala N., Yau C., 'A New Framework for Analyzing Test Generation and Diagnosis Algorithms for Wiring
Interconnects', IEEE proceedings of the International Test Conference, 1989, pp 63-70.

\section{Appendix A: The interconnection test patterns for the 64Mbit SDRAM circuit.}

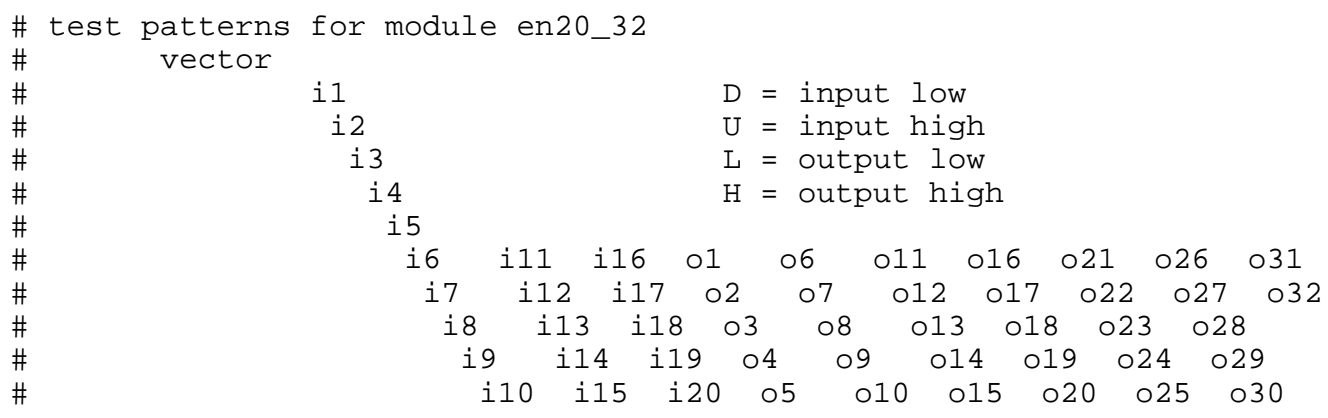

1

DDDDDDDDDDDDDDDDDDDDHHHHHнHнHнHнHнHнHнHнHнHнHнHнHнHн \# all-0 stimulus UDDDDDDDDDDDDDDDDDDDLLLLLLLLLLLLLLLLLLLHHHHHHHHHHHHH \# 20x walking-1 DUDDDDDDDDDDDDDDDDDDLHHHHHHHHHHHHHHHHHHLLLLLLLLLLLLL

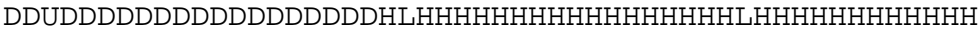

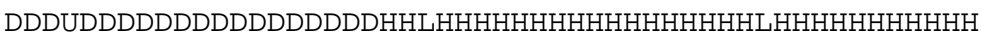

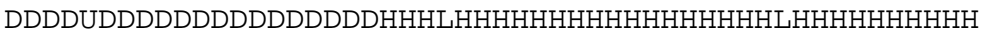

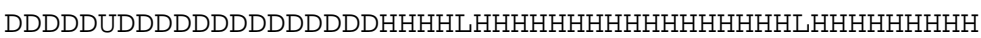

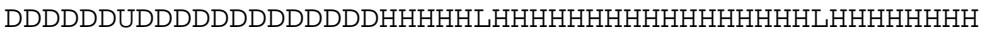

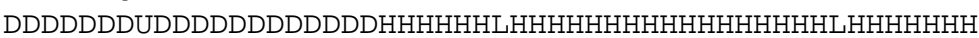

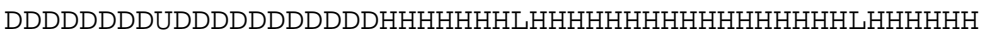

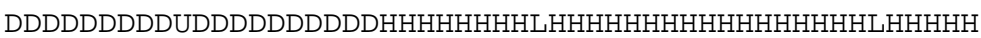

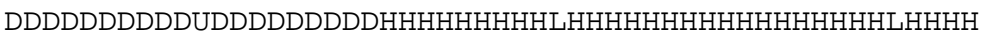

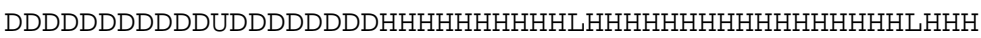

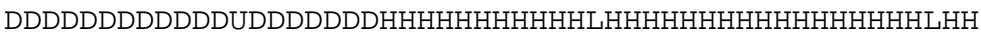

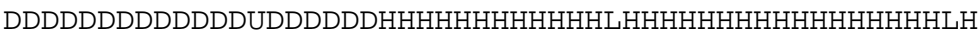
DDDDDDDDDDDDDDUDDDDDHHH H H H H H H H L L H H H H H H H H H H H H H L

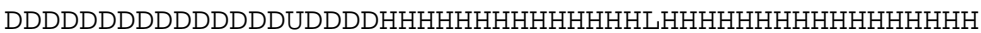

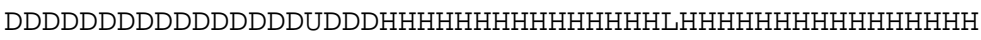

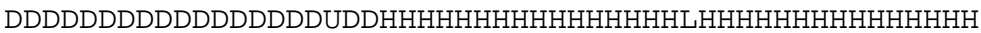

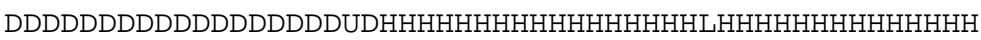

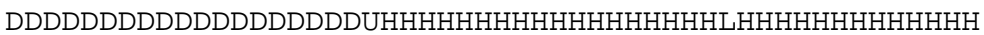
DUUUUUUUUUUUUUUUUUUULLLLLLLLLLLLLLLLLLLHНH HНHНH HНH H UDUUUUUUUUUUUUUUUUUULHнHнHнHнHнHнHнHHHHLLLLLLLLLLLLL \# 20x walking-0

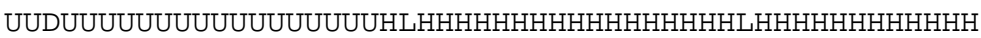

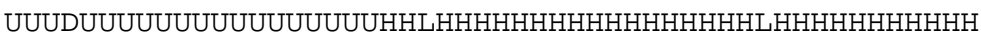
UUUUDUUUUUUUUUUUUUUUHHHLHHHH H H H H H H H H H H L L H H H H H H H H UUUUUDUUUUUUUUUUUUUUH H H L L H H H H H H H H H H H H H H H L UUUUUUDUUUUUUUUUUUUUH H H H L L H H H H H H H H H H H H H H UUUUUUUDUUUUUUUUUUUUHHH H H L H H H H H H H H H H H H H H L L H H H H H UUUUUUUUDUUUUUUUUUUUHH H H H H L L H H H H H H H H H H H H H H L H H H H H UUUUUUUUUDUUUUUUUUUUH H H H H H H L H H H H H H H H H H H H H H L UUUUUUUUUUDUUUUUUUUUH H H H H H H H L H H H H H H H H H H H H H H L UUUUUUUUUUUDUUUUUUUU H H H H H H H H H L H H H H H H H H H H H H H H UUUUUUUUUUUUDUUUUUUUH H H H H H H H H H L H H H H H H H H H H H H H H H L H UUUUUUUUUUUUUDUUUUUUH H H H H H H H H H L L UUUUUUUUUUUUUUDUUUUUH H H H H H H H H H H 2 L H H H H H H H H H H H H H H UUUUUUUUUUUUUUUDUUUUH H H H H H H H H H H H L L H H H H H H H H H H H H H H UUUUUUUUUUUUUUUUDUUUH H H H H H H H H H H H H L $H$ H H H H H H H H H H H H

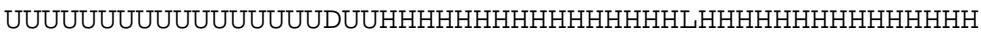

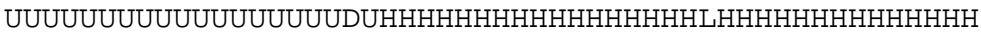
UUUUUUUUUUUUUUUUUUUD H H H H H H H H H H H H H H H H L 2 H H H H H H H H H H H

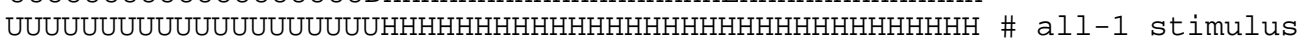


Appendix B: The Verilog description of the 64Mbit SDRAM SCITT implementation.

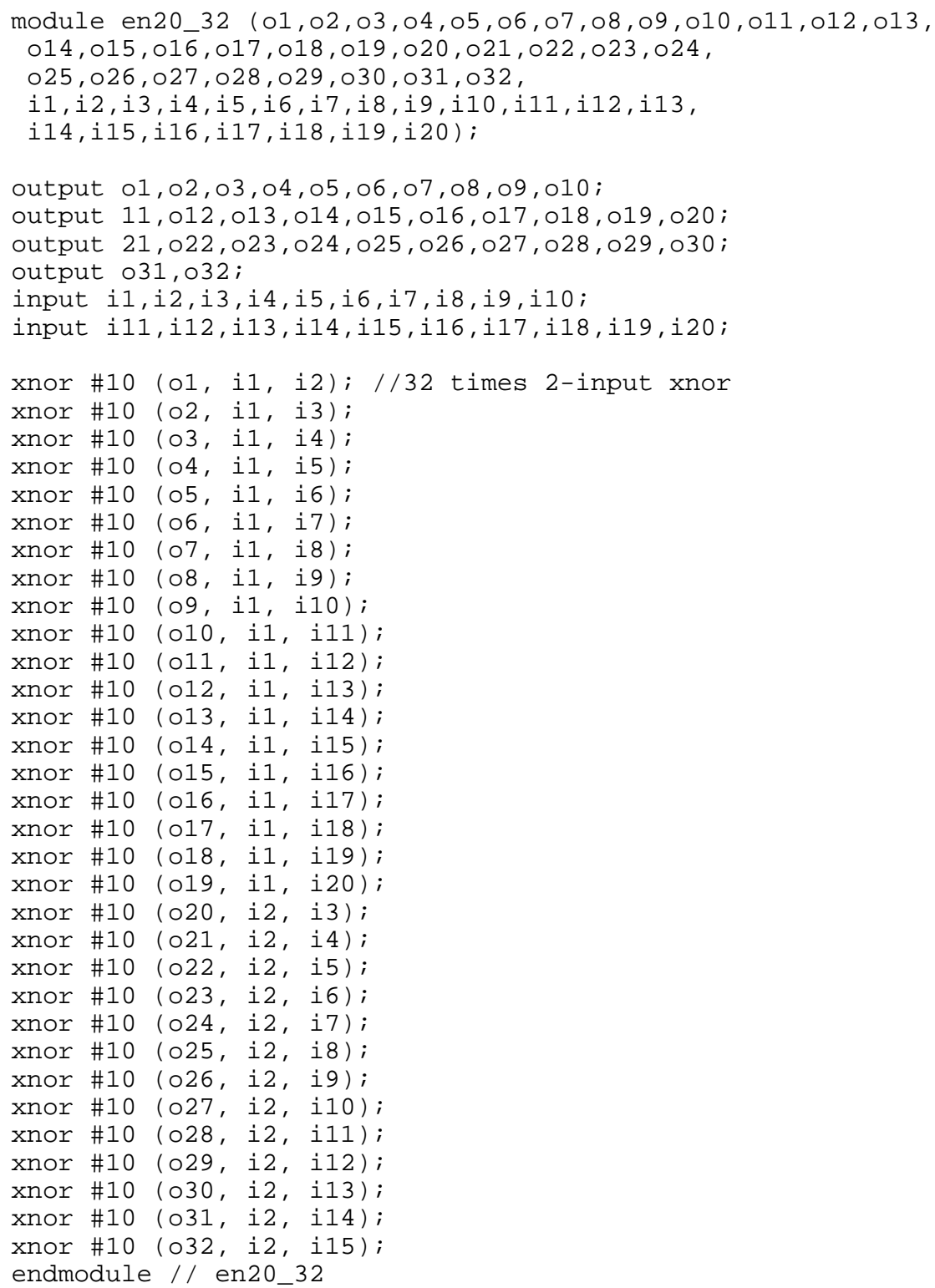

